\title{
medicines
}

ISSN 2305-6320

www.mdpi.com/journal/medicines

Article

\section{Potent and Synergistic Extract Combinations from Terminalia Catappa, Terminalia Mantaly and Monodora tenuifolia Against Pathogenic Yeasts}

\author{
Thierry Kammalac Ngouana ${ }^{1,2,3}$, Cedric Derick Jiatsa Mbouna ${ }^{1}$, \\ Rufin Marie Toghueo Kuipou ${ }^{1}$, Marthe Aimée Tchuente Tchuenmogne ${ }^{4}$, \\ Elisabeth Menkem Zeuko'’ ${ }^{1}$, Vincent Ngouana ${ }^{1}$, Michèle Mallié ${ }^{3}$, Sebastien Bertout ${ }^{3}$ and \\ Fabrice Fekam Boyom 1,*
}

1 Antimicrobial Agents Unit, Laboratory for Phytobiochemistry and Medicinal Plants Studies, Department of Biochemistry, Faculty of Science, University of Yaoundé I, P.O. Box 812, Messa-Yaoundé, Cameroon; E-Mails: ngouanathi@yahoo.com (T.K.N.); cedrickjiatsa@yahoo.com (C.D.J.M.); toghueo.rufin@yahoo.fr (R.M.T.K.); zeukoo@yahoo.com (E.M.Z.); vngouana@yahoo.fr (V.N.)

2 Clinical Biology Laboratory, Yaoundé Central Hospital, P.O. Box 87, Messa-Yaoundé, Cameroon

3 IRD UMI 233 TransVIHMI-UM INSERM U1175 TransVIHMI Laboratoire de Parasitologie et Mycologie Médicale, UFR Pharmacie, Université de Montpellier 1, 15, Avenue Charles Flahault_BP 1449134093 Montpellier, France; E-Mails: michele.mallie@univ-montp1.fr (M.M.); sebastien.bertout@univ-montp1.fr (S.B.)

4 Laboratory of Natural Products and Organic Synthesis, Department of Organic Chemistry, Faculty of Science, University of Yaoundé 1, P.O. Box 812, Messa-Yaoundé, Cameroon;

E-Mail: tch_aimee@yahoo.fr

* Author to whom correspondence should be addressed; E-Mail: fabrice.boyom@fulbrightmail.org; Tel.: +237-677-276-585.

Academic Editor: Ronan Batista

Received: 18 June 2015 / Accepted: 17 August 2015 / Published: 26 August 2015

\begin{abstract}
Mycoses caused by Candida and Cryptococcus species, associated with the advent of antifungal drug resistance have emerged as major health problems. Improved control measures and innovative therapies are needed. This paper describes results from the screening of bio-guided fractionated extracts alone and combinations of Terminalia catappa, Terminalia mantaly and Monodora tenuifolia harvested in Cameroon. Crude ethanolic, hydro-ethanolic and aqueous extracts and bio-guided fractions were screened for
\end{abstract}


antifungal activity against isolates of C. albicans, C. glabrata, C. parapsilosis and $C r$. neoformans and the reference strain C. albicans NR-29450. Minimal inhibitory concentrations (MIC) were determined using a broth micro dilution method according to the Clinical \& Laboratory Standards Institute (CLSI). Time kill kinetics of extracts alone and in combination were also evaluated. Extracts from T. mantaly stem bark were the most active with the best MIC values ranging from $0.04 \mathrm{mg} / \mathrm{mL}$ to $0.16 \mathrm{mg} / \mathrm{mL}$. Synergistic interactions were observed with combinations of sub-fractions from $M$. tenuifolia, T. mantaly and T. catappa. Combination of sub-fractions from M. tenuifolia and T. mantaly (C36/C12) showed synergistic interaction and fungicidal effect against four out of five tested yeasts. These results support further investigation of medicinal plant extracts alone and in combination as starting points for the development of alternative antifungal therapy.

Keywords: Terminalia catappa; Terminalia mantaly; Monodora tenuifolia; antifungal activity; bio-guided fractionation; combinations

\section{Introduction}

The knowledge of the epidemiology and pathogenesis of yeasts affecting humans has increased in the last three decades [1-4]. Many conditions have promoted the increase of opportunistic fungal infections in humans, especially candidiasis and cryptococcosis [1,3-12].

Candidiasis is the most common fungal infection in both immunocompromised and immunocompetent persons [10,13-17]. Invasive candidiasis is responsible for the high mortality among intensive care patients with a frequency of $40 \%-75 \%[3,9,15,18]$. C. albicans is the most frequent yeast involved in these infections, although other species are also frequently isolated such as C. parapsilosis, C. glabrata, C. tropicalis, C. krusei, C. lusitaniae, and C. dubliniensis. Furthermore, some other pathogenic yeasts are emerging, including C. famata, C. guilliermondii, C. kefyr and more recently C. africana [9,19]. Moreover, Cryptococcal meningitis caused by $C r$. neoformans is the most dangerous systemic mycosis associated with AIDS [20-22].

Fungal infections are treated using different families of drugs such as polyens, azoles, pyrimidine analogues, echinocandins and allylamines $[2,9,23]$. However, these drugs have some limitations such as high toxicity and low bioavailability (polyens) and reduced spectrum of action (echinocandins) [9,23]. Resistance has already been described for all these antifungal drugs and is more often associated with therapeutic failure [23-25]. These limitations in treatments associated with the increased knowledge of the epidemiology and pathogenicity of yeasts underline the urgent need for new antifungal agents with improved potency and innovative modes of action that could be further developed as antifungal treatments.

Natural products have proven efficacy in the treatment of a wide range of ailments. Ethnobotanically selected plants such as T. mantaly (Combretaceae), T. catappa (Combretaceae) and M. tenuifolia (Annonaceae) are frequently used in traditional medicine for the treatment of various infectious diseases [26,27]. This paper describes the antifungal activity of their extracts, fractions and sub-fractions against Candida spp and Cr. neoformans isolates. 


\section{Experimental Section}

\subsection{Collection of Plant Materials}

Organs from T. catappa, T. mantaly (Combretaceae) and M. tenuifolia (Annonaceae) were collected in the Yaoundé area and identified at the Cameroon National Herbarium where voucher specimens are kept under registration numbers 51244/HNC (Terminalia catappa Linné), 64212/HNC (Terminalia mantaly H. Perrier) and 30549/HNC (Monodora tenuifolia Benth). Leaves, twigs, fruits, fruits pericarp, pulp, seeds, seeds pericarp, branches and stem bark were collected and separately dried at room temperature and ground using a blender.

\subsection{Yeasts Isolates and Reference Strain}

Yeast isolates were provided by the laboratory of clinical biology, Yaoundé Central Hospital (Yaoundé, Cameroon), and consisted of clinical isolates of C. albicans, C. glabrata, C. parapsilosis and Cr. neoformans. C. albicans NR-29450 was obtained from BeiResources (Manassas, VA, USA) and used as positive control. These yeasts were maintained at room temperature and cultured at $37{ }^{\circ} \mathrm{C}$ for $24 \mathrm{~h}$ on Sabouraud Dextrose Agar (Oxoid) slants prior to use.

\subsection{Plant Extraction and Preliminary Screening of Antifungal Activity}

The crude extracts were prepared by maceration of individual plant material in each solvent; ethanol (95\%), distilled water and ethanol-water mixture $(70: 30, v / v)$, for $48 \mathrm{~h}$. Organic solvents were subsequently evaporated from filtrates using a rotary evaporator (Büchi 011, Flawil Switzerland). Water filtrates were dried using a desiccator at $60^{\circ} \mathrm{C}$ (Memmert UN30, Schwabach, Germany). Each plant powder was extracted separately three times using the same amount of solvent, and the dried extracts were pooled and weighed. The yields of extraction were calculated in percentage relative to the weight of the starting plant material.

For the preliminary antifungal screening, the crude extracts were dissolved in $10 \%$ dimethylsulfoxide (DMSO) and tested using the agar dilution method, according to the CLSI M44-A2 protocol [28,29] with some modifications. Briefly, sterile Mueller Hinton agar medium (Oxoid) supplemented with $2 \%$ glucose, was maintained at $45{ }^{\circ} \mathrm{C}$ and the extract added to obtain a final concentration of $40 \mathrm{mg} / \mathrm{mL}$. The mixture was then transferred in a Petri dish and let to solidify at room temperature. Plates were inoculated with $2.0 \times 10^{4}$ yeast cells $/ \mathrm{mL}$ in sterile normal saline solution and incubated at $37{ }^{\circ} \mathrm{C}$ for $48 \mathrm{~h}$. Growth control consisted of microorganisms cultured on the Mueller Hinton agar medium without plant extracts. Fluconazole (Sigma-Aldrich, Heidenheim, Germany) was used as positive control at $10 \mu \mathrm{g} / \mathrm{mL}$. Experiments were run simultaneously and each test performed in duplicate. At incubation time, yeast colonies were enumerated and inhibition percentages (IP) were calculated using the formula:

IP $(\%)=($ colonies in growth control - colonies in test dish $) \times 100 /$ colonies in growth control 
Crude extracts that showed an inhibition percentage of $\geq 75 \%$ against at least one of the tested yeasts were selected for the determination of minimal inhibitory concentration (MIC), defined as the lowest concentration at which there was no visible growth of the yeasts.

MIC values of the selected extracts were determined according to the CLSI M27-A3 protocol [30] with little modifications. Briefly, a serial two-fold dilution of each crude extract using Mueller Hinton broth medium (Oxoid) supplemented with $2 \%$ glucose was done in 96-wells microtiter plates starting with $40 \mathrm{mg} / \mathrm{mL}$ up to $0.04 \mathrm{mg} / \mathrm{mL}$. One hundred microliters of fungal inocula at the final concentrations of $0.5-2.5 \times 10^{3} \mathrm{CFU} / \mathrm{mL}$ were then added into each well of the plate to achieve a final volume of $200 \mu \mathrm{L}$. Plates were incubated at $37{ }^{\circ} \mathrm{C}$ for $48 \mathrm{~h}$. Fluconazole was tested as positive control at the highest concentration of $64 \mu \mathrm{g} / \mathrm{mL}$. MICs were determined based on turbidity in plate wells through macroscopic observation. Crude extracts that showed MIC $\leq 1.25 \mathrm{mg} / \mathrm{mL}$ against at least half of the tested yeasts were selected for progression to the bio-guided fractionation.

\subsection{Bio-Guided Fractionation of Selected Crude Extracts}

Extract fractionation consisted in partitioning $10 \mathrm{~g}$ of each selected extract in $300 \mathrm{~mL}$ mixture of methylene chloride/water $(v / v)$. After exhaustion of the methylene chloride phase, fractions were concentrated and dried. Change in MICs of the dried fractions was determined as described above and promising fractions were selected for progression. Five grams of each selected fraction was further partitioned into $150 \mathrm{~mL}$ mixture of hexane/methanol $(v / v)$ and the afforded sub-fractions tested for activity.

\subsection{Antifungal Activity of Combined Sub-Fractions}

Seven sub-fractions from the three plants were combined to give 11 interspecies combinations, with no combination between extracts from the same plant. Checkerboard tests were used to determine fractional inhibitory concentration indexes (FICIs) of combinations of sub-fractions against each test microorganism. The checkerboard broth microdilution method based on CLSI recommendations [31] consisted of diluting sub-fractions in the two directions of a 96-wells microplate. Mixed concentrations in wells ranged from $1 / 8 \times$ MIC to $8 \times$ MIC and $1 / 128 \times$ MIC to $8 \times$ MIC for the two sub-fractions, respectively. The fungal inoculum was added to give an ultimate concentration of $0.5-2.5 \times 10^{3} \mathrm{CFU} / \mathrm{mL}$ in a final volume of $100 \mu \mathrm{L}$. Plates were therefore incubated at $37{ }^{\circ} \mathrm{C}$ for $48 \mathrm{~h}$ and each test performed in duplicate.

Changes in the Fractional Inhibitory Concentration Indexes (FICIs) were calculated using the following formula, and the type of interaction was determined according to previously described criteria [31,32].

$\operatorname{FICI}_{(\mathrm{X} 1 / \mathrm{X} 2)}=\mathrm{MICX} 1$ in combination/MICX1 alone $+\mathrm{MICX}_{\mathrm{X} 2}$ in combination/MICX2 alone [33].

Based on these criteria, a combination was considered to be synergistic when the FICI was $\leq 0.5$, additive when it was $>0.5$ to $\leq 1$, indifferent when it was $>1.0$ to $\leq 4.0$. Antagonism was obtained with FICI $>4.0$. 


\subsection{Time Kill Kinetic Assay of the Most Active Combinations}

The time kill kinetic assay of the selected combinations was assessed using the method described by Klepser et al. [34] with some modifications. The assays were performed in duplicate in $1.5 \mathrm{~mL}$ conical tubes at final concentrations of 4xMIC for each sub-fraction in the Mueller Hinton broth. For each combination, three tubes were used, two containing sub-fractions alone and one containing the combination of sub-fractions. The final volume in each tube was $1 \mathrm{~mL}$, containing a fungal inoculum of $1-2.10^{5}$ cells $/ \mathrm{mL}$. After incubation under orbital shaking (IKA-Vibrax-VXR, Radnor, PA, USA) $(32 \times g)$ at $37^{\circ} \mathrm{C}$, and at time intervals of $0,2,4,6,8,12,16$ and $24 \mathrm{~h}$, a $50 \mu \mathrm{L}$ aliquot was collected from each culture to inoculate $950 \mu \mathrm{L}$ of sterile broth medium and incubated at $37{ }^{\circ} \mathrm{C}$ for $24 \mathrm{~h}$ to determine the number of cells $/ \mathrm{mL}$ using a Malassez haemocytometer (Thermo Fisher Scientific, Darmstadt, Germany). Time kill kinetic curves were plotted as log10 (number of viable cells) versus time. The following criteria were used to interpret the interactions: Synergy was obtained when the fungicidal effect led to $\geq 2 \log 10$ decrease in cells $/ \mathrm{mL}$ for the combination compared to the most active sub-fraction; additivity was defined as $<2 \log 10$ decrease in cells $/ \mathrm{mL}$ for the combination compared to the most active sub-fraction; indifference as $<2 \log 10$ increase in cells $/ \mathrm{mL}$ for the combination compared to the least active sub-fraction; and antagonism as $\geq 2 \log 10$ increase in cells $/ \mathrm{mL}$ for the combination compared to the least active sub-fraction [9,34].

\subsection{Phytochemical Screening of Selected Crude Extracts and Sub-Fractions}

A qualitative phytochemical analysis of promising extracts and sub-fractions was carried out to identify groups of secondary metabolites known as having antifungal activity, including flavonoids, tannins, anthraquinones, alkaloids, steroids, glucosides, saponins, triterpenes, anthocyanins and phenols and using the previously described protocols [35-38].

\section{Results}

\subsection{Plant Extraction and Preliminary Screening of Antifungal Activity}

Thirty-four crude extracts were obtained with yields ranging from $1.7 \%-36.7 \%$ relative to the weights of the starting plant materials. Results from the preliminary screening of antifungal activity enabled the selection of 23 extracts based on their inhibitory percentages against clinical isolates and the reference Candida albicans NR-29450 ( $\geq 75 \%$ against at least one of the tested yeasts). Change in MIC values in liquid medium ranging from $0.04 \mathrm{mg} / \mathrm{mL}$ to $40.0 \mathrm{mg} / \mathrm{mL}$ was observed as described in Table 1. Based on our selection criteria (MIC $\leq 1.25 \mathrm{mg} / \mathrm{mL}$ against at least half of tested yeasts), 15 crude extracts were selected and progressed to the bio-guided fractionation, including extracts A1 to A15. 
Table 1. Minimal inhibitory concentrations (MIC) of crude extracts, fractions and sub-fractions.

\begin{tabular}{|c|c|c|c|c|c|c|}
\hline \multirow{3}{*}{ Codes } & \multirow{3}{*}{ Crude Extracts } & \multicolumn{5}{|c|}{${ }^{\mathrm{a}} \mathrm{MIC}(\mathrm{mg} / \mathrm{mL})$} \\
\hline & & \multirow{2}{*}{$\begin{array}{c}\text { Reference Strain } \\
\text { C. albicans NR-29450 }\end{array}$} & \multicolumn{4}{|c|}{ Isolates } \\
\hline & & & C. albicans & C. glabrata & C. parapsilosis & Cr. neoformans \\
\hline A1 & $\mathrm{Te} \mathrm{C} \mathrm{L} \mathrm{H} \mathrm{H}_{2} \mathrm{O}$ & 0.31 & 2.50 & 0.63 & 2.50 & 0.63 \\
\hline $\mathrm{A} 2$ & $\mathrm{Te} \mathrm{C} \mathrm{L} \mathrm{H}_{2} \mathrm{O} / \mathrm{EthOH}$ & 0.08 & 0.31 & 0.08 & 0.31 & 0.16 \\
\hline $\mathrm{A} 3$ & Te C L EthOH & 0.31 & 0.16 & 0.08 & 0.31 & 0.31 \\
\hline A4 & $\mathrm{Te} \mathrm{C} \mathrm{Sb} \mathrm{H}_{2} \mathrm{O}$ & 0.16 & 0.63 & 0.31 & 0.31 & 0.63 \\
\hline A5 & $\mathrm{Te} \mathrm{C} \mathrm{Sb} \mathrm{H}_{2} \mathrm{O} / \mathrm{EthOH}$ & 0.08 & 2.50 & 0.63 & 0.31 & 0.63 \\
\hline A6 & Te C Sb EthOH & 0.16 & 0.16 & 0.16 & 0.16 & 0.16 \\
\hline A7 & Te $\mathrm{M} \mathrm{L} \mathrm{H}_{2} \mathrm{O}$ & 0.31 & 2.50 & 0.31 & 0.31 & 0.16 \\
\hline A8 & Te M L H $\mathrm{H}_{2} \mathrm{O} /$ EthOH & 0.04 & 0.04 & 0.04 & 0.08 & 0.08 \\
\hline A9 & Te M L EthOH & 0.04 & 0.08 & 0.08 & 0.16 & 0.16 \\
\hline A10 & $\mathrm{Te} \mathrm{M} \mathrm{Sb}_{2} \mathrm{O}$ & 0.08 & 0.08 & 0.08 & 0.08 & 0.08 \\
\hline A11 & $\mathrm{Te} \mathrm{M} \mathrm{Sb} \mathrm{H}_{2} \mathrm{O} / \mathrm{EthOH}$ & 0.08 & 0.31 & 0.16 & 0.16 & 0.08 \\
\hline $\mathrm{A} 12$ & Te M Sb EthOH & 0.08 & 0.16 & 0.16 & 0.08 & 0.04 \\
\hline $\mathrm{A} 13$ & Mo T L H $\mathrm{H}_{2} \mathrm{O}$ & 10.00 & 1.25 & 0.63 & 10.00 & 1.25 \\
\hline A14 & Mo T L $\mathrm{H}_{2} \mathrm{O} /$ EthOH & 5.00 & 0.63 & 0.63 & 1.25 & 1.25 \\
\hline A15 & Mo T L EthOH & 2.50 & 5.00 & 1.25 & 1.25 & 0.08 \\
\hline A16 & Mo T Tw $\mathrm{H}_{2} \mathrm{O}$ & 20.00 & 10.00 & 5.00 & 5.00 & 2.50 \\
\hline A17 & Mo $\mathrm{T} \mathrm{Br} \mathrm{H}_{2} \mathrm{O}$ & 10.00 & 5.00 & 5.00 & 2.50 & 5.00 \\
\hline A18 & $\mathrm{Mo} \mathrm{T} \mathrm{Pu} \mathrm{H}_{2} \mathrm{O}$ & 2.50 & 10.00 & 5.00 & 5.00 & 10.00 \\
\hline A19 & Mo T Pu $\mathrm{H}_{2} \mathrm{O} / \mathrm{EthOH}$ & 5.00 & 5.00 & 5.00 & 10.00 & 2.50 \\
\hline $\mathrm{A} 20$ & Mo T Pu EthOH & 5.00 & 2.50 & 5.00 & 10.00 & 2.50 \\
\hline $\mathrm{A} 21$ & Mo T Se $\mathrm{H}_{2} \mathrm{O} / \mathrm{EthOH}$ & 40.00 & 10.00 & 10.00 & 40.00 & 40.00 \\
\hline $\mathrm{A} 22$ & Mo T FrPe EthOH & 10.00 & 5.00 & 5.00 & 10.00 & 2.50 \\
\hline $\mathrm{A} 23$ & Mo T PSe EthOH & 20.00 & 10.00 & 20.00 & 40.00 & 20.00 \\
\hline
\end{tabular}


Table 1. Cont.

\begin{tabular}{|c|c|c|c|c|c|c|c|}
\hline \multirow{3}{*}{ Codes } & \multirow{3}{*}{ Fractions } & \multicolumn{6}{|c|}{${ }^{\mathrm{a}} \mathrm{MIC}(\mathrm{mg} / \mathrm{mL})$} \\
\hline & & \multirow{2}{*}{\multicolumn{2}{|c|}{$\begin{array}{c}\text { Reference Strain } \\
\text { C. albicans NR-29450 }\end{array}$}} & \multicolumn{4}{|c|}{ Isolates } \\
\hline & & & & C. albicans & C. glabrata & C. parapsilosis & Cr. neoformans \\
\hline B1 & \multirow{2}{*}{$\mathrm{Te} C \mathrm{~L} \mathrm{H}_{2} \mathrm{O}$} & $\mathrm{Fr} \mathrm{H}_{2} \mathrm{O}$ & 1.25 & 1.25 & 5 & 0.63 & 0.16 \\
\hline B2 & & $\mathrm{Fr} \mathrm{CH}{ }_{2} \mathrm{Cl}_{2}$ & 0.63 & 1.25 & 5 & 0.16 & 0.16 \\
\hline B3 & \multirow{2}{*}{$\mathrm{Te} \mathrm{C} \mathrm{L} \mathrm{H} \mathrm{H}_{2} \mathrm{O} / \mathrm{EthOH}$} & Fr $\mathrm{H}_{2} \mathrm{O}$ & 0.31 & 2.5 & 0.63 & 0.31 & 0.31 \\
\hline B4 & & $\mathrm{Fr} \mathrm{CH}{ }_{2} \mathrm{Cl}_{2}$ & 0.31 & 5 & 0.63 & 0.31 & 0.31 \\
\hline B5 & \multirow{2}{*}{ Te C L EthOH } & Fr $\mathrm{H}_{2} \mathrm{O}$ & 0.63 & $>5.000$ & 0.63 & 0.31 & 0.16 \\
\hline B6 & & $\mathrm{Fr} \mathrm{CH} \mathrm{Cl}_{2}$ & 0.63 & 5 & 0.63 & 0.63 & 0.31 \\
\hline B7 & \multirow{2}{*}{$\mathrm{Te} \mathrm{C} \mathrm{Sb} \mathrm{H}_{2} \mathrm{O}$} & Fr $\mathrm{H}_{2} \mathrm{O}$ & 1.25 & 2.5 & 2.5 & 0.63 & 0.31 \\
\hline B8 & & Fr $\mathrm{CH}_{2} \mathrm{Cl}_{2}$ & 1.25 & 5 & 5 & 2.5 & 0.63 \\
\hline B9 & \multirow{2}{*}{$\mathrm{Te} \mathrm{C} \mathrm{Sb} \mathrm{H}_{2} \mathrm{O} / \mathrm{EthOH}$} & Fr $\mathrm{H}_{2} \mathrm{O}$ & 0.31 & 5 & 2.5 & 2.5 & 0.63 \\
\hline B10 & & $\mathrm{Fr} \mathrm{CH} \mathrm{Cl}_{2}$ & 0.16 & 0.63 & 1.25 & 1.25 & 0.63 \\
\hline B11 & \multirow{2}{*}{ Te C Sb EthOH } & Fr $\mathrm{H}_{2} \mathrm{O}$ & 0.16 & 0.63 & 2.5 & 1.25 & 0.63 \\
\hline B12 & & $\mathrm{Fr} \mathrm{CH} \mathrm{Cl}_{2}$ & 0.31 & $>5.000$ & 5 & 5 & 1.25 \\
\hline B13 & \multirow{2}{*}{$\mathrm{Te} \mathrm{M} \mathrm{L} \mathrm{H}_{2} \mathrm{O}$} & Fr $\mathrm{H}_{2} \mathrm{O}$ & 2.5 & 1.25 & 0.31 & 0.31 & 0.16 \\
\hline B14 & & $\mathrm{Fr} \mathrm{CH}{ }_{2} \mathrm{Cl}_{2}$ & 2.5 & 0.63 & 0.16 & 0.08 & 0.08 \\
\hline B15 & \multirow{2}{*}{$\mathrm{Te} \mathrm{M} \mathrm{L} \mathrm{H}_{2} \mathrm{O} / \mathrm{EthOH}$} & Fr $\mathrm{H}_{2} \mathrm{O}$ & 2.5 & 1.25 & 0.31 & 0.16 & 0.04 \\
\hline B16 & & $\mathrm{Fr} \mathrm{CH}{ }_{2} \mathrm{Cl}_{2}$ & 2.5 & 0.16 & 0.31 & 0.16 & 0.08 \\
\hline B17 & \multirow{2}{*}{ Te M L EthOH } & Fr $\mathrm{H}_{2} \mathrm{O}$ & 2.5 & 0.63 & 0.63 & 0.31 & 0.31 \\
\hline B18 & & $\mathrm{Fr} \mathrm{CH} \mathrm{Cl}_{2}$ & 0.63 & 0.31 & 0.08 & 0.04 & 0.04 \\
\hline B19 & \multirow{2}{*}{$\mathrm{Te} \mathrm{M} \mathrm{Sb} \mathrm{H}_{2} \mathrm{O}$} & Fr $\mathrm{H}_{2} \mathrm{O}$ & 1.25 & 0.63 & 0.16 & 0.08 & 0.08 \\
\hline B20 & & Fr $\mathrm{CH}_{2} \mathrm{Cl}_{2}$ & 0.63 & 0.31 & 0.16 & 0.16 & 0.08 \\
\hline B21 & \multirow{2}{*}{$\mathrm{Te} \mathrm{M} \mathrm{Sb} \mathrm{H}_{2} \mathrm{O} / \mathrm{EthOH}$} & Fr $\mathrm{H}_{2} \mathrm{O}$ & 0.31 & 1.25 & 0.31 & 0.31 & 0.16 \\
\hline $\mathrm{B} 22$ & & $\mathrm{Fr} \mathrm{CH} \mathrm{Cl}_{2}$ & 0.63 & 1.25 & 0.63 & 0.31 & 0.31 \\
\hline
\end{tabular}


Table 1. Cont.

\begin{tabular}{|c|c|c|c|c|c|c|c|}
\hline \multirow{3}{*}{ Codes } & \multirow{3}{*}{ Fractions } & \multicolumn{6}{|c|}{${ }^{\mathrm{a}} \mathrm{MIC}(\mathrm{mg} / \mathrm{mL})$} \\
\hline & & \multirow{2}{*}{\multicolumn{2}{|c|}{$\begin{array}{c}\text { Reference Strain } \\
\text { C. albicans NR-29450 }\end{array}$}} & \multicolumn{4}{|c|}{ Isolates } \\
\hline & & & & C. albicans & C. glabrata & C.parapsilosis & Cr. neoformans \\
\hline B23 & \multirow{2}{*}{ Te M Sb EthOH } & Fr $\mathrm{H}_{2} \mathrm{O}$ & 0.63 & 1.25 & 0.31 & 0.16 & 0.16 \\
\hline B24 & & $\mathrm{Fr} \mathrm{CH}{ }_{2} \mathrm{Cl}_{2}$ & 1.25 & 1.25 & 0.31 & 0.16 & 0.16 \\
\hline B25 & \multirow{2}{*}{ Mo T L $\mathrm{H}_{2} \mathrm{O}$} & $\mathrm{Fr} \mathrm{H}_{2} \mathrm{O}$ & 5 & 5 & 0.08 & $>5.000$ & $>5.000$ \\
\hline B26 & & $\mathrm{Fr} \mathrm{CH}{ }_{2} \mathrm{Cl}_{2}$ & 2.5 & 2.5 & 0.63 & 2.5 & 2.5 \\
\hline B27 & \multirow{2}{*}{ Mo T L $\mathrm{H}_{2} \mathrm{O} / \mathrm{EthOH}$} & $\mathrm{Fr} \mathrm{H}_{2} \mathrm{O}$ & 2.5 & 5 & 5 & 5 & 2.5 \\
\hline B28 & & $\mathrm{Fr} \mathrm{CH}{ }_{2} \mathrm{Cl}_{2}$ & 2.5 & 2.5 & 1.25 & 0.63 & 0.31 \\
\hline B29 & \multirow{2}{*}{ Mo T L EthOH } & $\mathrm{Fr} \mathrm{H}_{2} \mathrm{O}$ & 2.5 & $>5.000$ & 2.5 & 5 & 5 \\
\hline $\mathrm{B} 30$ & & $\mathrm{Fr} \mathrm{CH}{ }_{2} \mathrm{Cl}_{2}$ & 2.5 & 2.5 & 0.63 & 0.63 & 0.63 \\
\hline \multirow{3}{*}{ Codes } & \multirow{3}{*}{ Sub-fractions } & \multicolumn{6}{|c|}{${ }^{\mathrm{a}} \mathrm{MIC}(\mathrm{mg} / \mathrm{mL})$} \\
\hline & & \multicolumn{2}{|c|}{ Reference Strain } & \multicolumn{4}{|c|}{ Isolates } \\
\hline & & \multicolumn{2}{|c|}{ C. albicans NR-29450 } & C. albicans & C. glabrata & C.parapsilosis & Cr. neoformans \\
\hline $\mathrm{C} 2$ & Te C L H $\mathrm{H}_{2} \mathrm{O} /$ EthOH Fr $\mathrm{H}_{2} \mathrm{O}$ & $\mathrm{sFr} \mathrm{CH}_{3} \mathrm{OH}$ & 0.31 & 0.62 & 0.31 & 0.31 & 0.16 \\
\hline $\mathrm{C} 4$ & Te C L H${ }_{2} \mathrm{O} /$ EthOH Fr CH $2 \mathrm{Cl}_{2}$ & $\mathrm{sFr} \mathrm{CH}_{3} \mathrm{OH}$ & 0.31 & 0.62 & 0.31 & 0.62 & 0.31 \\
\hline $\mathrm{C} 5$ & \multirow{2}{*}{ Te C L EthOH Fr $\mathrm{CH}_{2} \mathrm{Cl}_{2}$} & sFr $\mathrm{C}_{6} \mathrm{H}_{12}$ & 0.62 & 1.25 & 0.62 & 1.25 & 0.62 \\
\hline C6 & & $\mathrm{sFr} \mathrm{CH}_{3} \mathrm{OH}$ & 1.25 & 1.25 & 1.25 & $>1.25$ & 1.25 \\
\hline $\mathrm{C} 8$ & Te C Sb $\mathrm{H}_{2} \mathrm{O} / \mathrm{EthOH}$ Fr $\mathrm{CH}_{2} \mathrm{Cl}_{2}$ & $\mathrm{sFr} \mathrm{CH}_{3} \mathrm{OH}$ & 0.16 & 1.25 & 0.31 & 0.62 & 0.08 \\
\hline $\mathrm{C} 10$ & $\mathrm{Te} \mathrm{M} \mathrm{L} \mathrm{H}_{2} \mathrm{O}$ Fr $\mathrm{H}_{2} \mathrm{O}$ & $\mathrm{sFr} \mathrm{CH}_{3} \mathrm{OH}$ & 0.16 & 0.62 & 0.31 & 0.31 & 0.16 \\
\hline $\mathrm{C} 12$ & Te M L $\mathrm{H}_{2} \mathrm{O}$ Fr $\mathrm{CH}_{2} \mathrm{Cl}_{2}$ & $\mathrm{sFr} \mathrm{CH}_{3} \mathrm{OH}$ & 0.31 & 0.62 & 0.08 & 0.08 & 0.08 \\
\hline C14 & Te M L $\mathrm{H}_{2} \mathrm{O} /$ EthOH Fr $\mathrm{H}_{2} \mathrm{O}$ & $\mathrm{sFr} \mathrm{CH}_{3} \mathrm{OH}$ & 0.31 & 0.62 & 0.16 & 0.16 & 0.16 \\
\hline $\mathrm{C} 15$ & \multirow{2}{*}{ Te M L H ${ }_{2} \mathrm{O} /$ EthOH $\mathrm{FrCH}_{2} \mathrm{Cl}_{2}$} & $\mathrm{sFr} \mathrm{C}_{6} \mathrm{H}_{12}$ & 0.31 & 0.31 & 0.31 & 0.31 & 0.16 \\
\hline $\mathrm{C} 16$ & & $\mathrm{sFr} \mathrm{CH}_{3} \mathrm{OH}$ & 0.16 & 0.16 & 0.16 & 0.62 & 0.16 \\
\hline $\mathrm{C} 18$ & Te M L EthOH Fr $\mathrm{H}_{2} \mathrm{O}$ & $\mathrm{sFr} \mathrm{CH}_{3} \mathrm{OH}$ & 0.31 & 0.16 & 0.31 & 0.31 & 0.62 \\
\hline $\mathrm{C} 20$ & Te M L EthOH Fr $\mathrm{CH}_{2} \mathrm{Cl}_{2}$ & $\mathrm{sFr} \mathrm{CH}_{3} \mathrm{OH}$ & 0.16 & 0.16 & 0.31 & 0.31 & 0.31 \\
\hline $\mathrm{C} 22$ & $\mathrm{Te} \mathrm{M} \mathrm{Sb} \mathrm{H}_{2} \mathrm{O}$ Fr $\mathrm{H}_{2} \mathrm{O}$ & $\mathrm{sFr} \mathrm{CH} \mathrm{CH}_{3} \mathrm{OH}$ & 0.16 & 0.08 & 0.08 & 0.16 & 0.04 \\
\hline
\end{tabular}


Table 1. Cont.

\begin{tabular}{|c|c|c|c|c|c|c|c|}
\hline \multirow{3}{*}{ Codes } & \multirow{3}{*}{ Sub-fractions } & \multicolumn{6}{|c|}{${ }^{\mathrm{a}} \mathrm{MIC}(\mathrm{mg} / \mathrm{mL})$} \\
\hline & & \multirow{2}{*}{\multicolumn{2}{|c|}{$\begin{array}{c}\text { Reference Strain } \\
\text { C. albicans NR-29450 }\end{array}$}} & \multicolumn{4}{|c|}{ Isolates } \\
\hline & & & & \multirow{2}{*}{$\frac{\text { C. albicans }}{0.08}$} & \multirow{2}{*}{$\frac{\text { C. glabrata }}{0.08}$} & \multirow{2}{*}{$\frac{\text { C.parapsilosis }}{0.16}$} & \multirow{2}{*}{$\frac{\text { Cr. neoformans }}{0.04}$} \\
\hline $\mathrm{C} 24$ & $\mathrm{Te} \mathrm{M} \mathrm{Sb} \mathrm{H}_{2} \mathrm{O} \mathrm{Fr} \mathrm{CH}_{2} \mathrm{Cl}_{2}$ & $\mathrm{sFr} \mathrm{CH}_{3} \mathrm{OH}$ & 0.16 & & & & \\
\hline $\mathrm{C} 26$ & 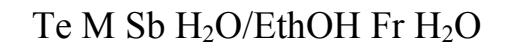 & $\mathrm{sFr} \mathrm{CH}_{3} \mathrm{OH}$ & 0.04 & 0.08 & 0.08 & 0.16 & 0.08 \\
\hline $\mathrm{C} 28$ & $\mathrm{Te} \mathrm{M} \mathrm{Sb} \mathrm{H}{ }_{2} \mathrm{O} / \mathrm{EthOH} \mathrm{FrCH}_{2} \mathrm{Cl}_{2}$ & sFr $\mathrm{CH}_{3} \mathrm{OH}$ & 0.31 & 1.25 & 1.25 & 1.25 & 0.62 \\
\hline $\mathrm{C} 30$ & $\mathrm{Te} \mathrm{M} \mathrm{Sb}$ EthOH Fr $\mathrm{H}_{2} \mathrm{O}$ & sFr $\mathrm{CH}_{3} \mathrm{OH}$ & 0.31 & 0.31 & 0.31 & 0.31 & 0.31 \\
\hline $\mathrm{C} 32$ & Te M Sb EthOH Fr $\mathrm{CH}_{2} \mathrm{Cl}_{2}$ & $\mathrm{sFr} \mathrm{CH}_{3} \mathrm{OH}$ & 0.16 & 0.08 & 0.08 & 0.16 & 0.08 \\
\hline C33 & \multirow{2}{*}{ Mo $\mathrm{T} \mathrm{L} \mathrm{H}{ }_{2} \mathrm{O} /$ EthOH Fr $\mathrm{CH}_{2} \mathrm{Cl}_{2}$} & $\mathrm{sFr} \mathrm{C}_{6} \mathrm{H}_{12}$ & $>1.25$ & $>1.25$ & $>1.25$ & $>1.25$ & $>1.25$ \\
\hline $\mathrm{C} 34$ & & $\mathrm{sFr} \mathrm{CH}_{3} \mathrm{OH}$ & 1.25 & 1.25 & 1.25 & 1.25 & $>1.25$ \\
\hline $\mathrm{C} 35$ & \multirow{2}{*}{ Mo T L EthOH Fr $\mathrm{CH}_{2} \mathrm{Cl}_{2}$} & $\mathrm{sFr} \mathrm{C}_{6} \mathrm{H}_{12}$ & 1.25 & $>1.25$ & $>1.25$ & $>1.25$ & 1.25 \\
\hline \multirow[t]{2}{*}{$\mathrm{C} 36$} & & $\mathrm{sFr} \mathrm{CH}_{3} \mathrm{OH}$ & 1.25 & 0.62 & 0.62 & $>1.25$ & 1.25 \\
\hline & Fluconazole $(\mu \mathrm{g} / \mathrm{mL})$ & & 0.50 & 1.00 & 8.00 & 8.00 & 2.00 \\
\hline
\end{tabular}

${ }^{a}$ Minimal Inhibitory Concentration (lowest concentration at which there is no visible growth of yeast); Mo T: Monodora tenuifolia; Te C: Terminalia catappa; Te M: Terminalia mantaly; L: leaves; Sb: stem bark; Tw: twigs; Br: branches; Pu: pulp; Se: seeds; PSe: seeds pericarp; FrPe: fruit pericarp; $\mathrm{H}_{2} \mathrm{O}$ : distilled water; $\mathrm{H}_{2} \mathrm{O}$ /EthOH: hydroethanol; EthOH: ethanol; $\mathrm{Fr} \mathrm{H}_{2} \mathrm{O}$ : aqueous fraction; $\mathrm{Fr} \mathrm{CH}_{2} \mathrm{Cl}_{2}$ : methylene chloride fraction; $\mathrm{sFr} \mathrm{C}_{6} \mathrm{H}_{12}$ : Hexanic sub-fraction; sFr $\mathrm{CH}_{3} \mathrm{OH}$ : Methanolic sub-fraction. A1-A23: crude extracts; B1-B30: fractions; $\mathrm{C} 2-\mathrm{C} 36$ : sub-fractions. 


\subsection{Activity of Bio-Guided Fractions}

The first partition of the 15 selected extracts generated 30 fractions out of which 18 were progressed based on their MIC values (median MIC $\leq 0.62 \mathrm{mg} / \mathrm{mL}$ ), including B3-B6, B10, B13-B24, and B28. Subsequent to the partition of the 18 fractions, seven sub-fractions (C2, C12, C22, C24, C26, C32 and C36) were afforded with median $\mathrm{MIC} \leq 0.31 \mathrm{mg} / \mathrm{mL}$. The most active sub-fraction (C26) showed a median MIC of $0.08 \mathrm{mg} / \mathrm{mL}$. MICs of fractions and sub-fractions are summarized in Table 2.

Globally, there was a significant increase in activity from crude extracts to sub-fractions, with median MICs $=0.31 \mathrm{mg} / \mathrm{mL}$ for crude extracts; median MICs $=0.31 \mathrm{mg} / \mathrm{mL}$ for fractions; and median MICs $=0.08 \mathrm{mg} / \mathrm{mL}$ for sub-fractions. Extracts from T. mantaly showed more potency, while those from $M$. tenuifolia were the least active against the tested yeasts. There was no correlation between observed activities and solvents used for extraction.

Table 2. Effect of combined sub-fractions on the tested yeasts.

\begin{tabular}{|c|c|c|c|c|c|c|c|c|c|c|}
\hline \multirow{2}{*}{$\begin{array}{c}\text { Combinations } \\
\text { of } \\
\text { sub-fractions }\end{array}$} & \multicolumn{2}{|c|}{$\begin{array}{c}\text { C. albicans NR- } \\
29450\end{array}$} & \multicolumn{2}{|c|}{ C. albicans } & \multicolumn{2}{|c|}{ C. glabrata } & \multicolumn{2}{|c|}{$\begin{array}{c}C . \\
\text { parapsilosis }\end{array}$} & \multicolumn{2}{|c|}{ Cr. neoformans } \\
\hline & FICI & Int & FICI & Int & FICI & Int & FICI & Int & FICI & Int \\
\hline $\mathrm{C} 2 / \mathrm{C} 12$ & 1.06 & I & 1.06 & I & 1.06 & I & 1.00 & $\mathrm{~A}$ & 0.62 & $\mathrm{~A}$ \\
\hline $\mathrm{C} 2 / \mathrm{C} 22$ & 0.75 & A & 0.50 & S & 0.75 & A & 0.62 & $\mathrm{~A}$ & 0.31 & S \\
\hline $\mathrm{C} 2 / \mathrm{C} 24$ & 0.56 & $\mathrm{~A}$ & 0.75 & A & 0.75 & A & 0.53 & $\mathrm{~A}$ & 0.31 & S \\
\hline $\mathrm{C} 2 / \mathrm{C} 26$ & 0.62 & $\mathrm{~A}$ & 0.75 & A & 0.75 & A & 1.12 & I & 0.62 & A \\
\hline $\mathrm{C} 2 / \mathrm{C} 32$ & 0.56 & A & 1.12 & I & 0.75 & A & 1.00 & A & 0.37 & S \\
\hline $\mathrm{C} 36 / \mathrm{C} 2$ & 0.50 & $\mathrm{~A}$ & 0.62 & A & 0.50 & S & 0.50 & S & 0.50 & S \\
\hline $\mathrm{C} 36 / \mathrm{C} 12$ & 0.37 & $\mathrm{~S}$ & 0.62 & A & 0.56 & A & 0.37 & S & 0.25 & S \\
\hline $\mathrm{C} 36 / \mathrm{C} 22$ & 0.53 & A & 0.62 & A & 0.75 & A & 0.75 & $\mathrm{~A}$ & 0.37 & S \\
\hline $\mathrm{C} 36 / \mathrm{C} 24$ & 1.00 & $\mathrm{~S}$ & 1.12 & I & 0.50 & S & 0.75 & A & 0.50 & S \\
\hline $\mathrm{C} 36 / \mathrm{C} 26$ & 0.75 & A & 0.75 & A & 0.62 & A & 0.56 & $\mathrm{~A}$ & 0.25 & S \\
\hline $\mathrm{C} 36 / \mathrm{C} 32$ & 0.62 & $\mathrm{~S}$ & 0.50 & $\mathrm{~S}$ & 0.75 & A & 0.56 & A & 0.62 & A \\
\hline
\end{tabular}

FICI: Fractional Inhibitory Concentration Index; Int: interaction; I: indifference; A: additivity; S: synergy; C2: Te CL H $\mathrm{H}_{2} \mathrm{O} / \mathrm{EthOH}$ Fr $\mathrm{H}_{2} \mathrm{O}$ sFr CH $\mathrm{CH}_{3} \mathrm{OH}$; C12: Te ML $\mathrm{H}_{2} \mathrm{O}$ Fr CH $\mathrm{Cl}_{2} \mathrm{Cl}_{2}$ sFr CH $\mathrm{CH}_{3} \mathrm{OH}$; C22: Te MSb H $\mathrm{H}_{2} \mathrm{O}$ Fr $\mathrm{H}_{2} \mathrm{O}$ sFr CH 33 OH; C24: Te MSb $\mathrm{H}_{2} \mathrm{O}$ Fr CH $\mathrm{CH}_{2} \mathrm{Cl}_{2} \mathrm{sFr} \mathrm{CH} \mathrm{CH}_{3} \mathrm{OH}$; C26: Te $\mathrm{MSbH}_{2} \mathrm{O} / \mathrm{EthOH} \mathrm{Fr} \mathrm{H}_{2} \mathrm{O}$ sFr CH $\mathrm{CH}_{3} \mathrm{OH}$; C32: Te MSb EthOH Fr $\mathrm{CH}_{2} \mathrm{Cl}_{2}$ sFr CH 3 OH; C36: Mo TL EthOH Fr $\mathrm{CH}_{2} \mathrm{Cl}_{2} \mathrm{sFr} \mathrm{CH} \mathrm{CH}_{3} \mathrm{OH}$.

\subsection{Activity of Combined Sub-Fractions}

Eleven combinations were prepared from seven selected sub-fractions (C2, C12, C22, C24, C26, $\mathrm{C} 32$ and C36) and tested for their bioactivity. The seven sub-fractions used consisted of five from T. mantaly, one from T. catappa and one from M. tenuifolia. All the calculated fractional inhibitory concentrations (FICIs) showed values between 0.25 and 1.12, presenting synergy, additivity or indifference (Table 2). No case of antagonism was observed. Two combinations (C36/C2 and C36/C12) exerted above 50\% synergistic interaction associated with more than $75 \%$ of MIC reduction. These combinations were prepared from a M. tenuifolia sub-fraction (C36) associated with each sub-fraction of T. mantaly $(\mathrm{C} 12)$ and $T$. catappa $(\mathrm{C} 2)$, respectively. They were therefore submitted to time kill kinetic assays. 


\subsection{Time Kill Kinetics of the Promising Sub-Fractions and Their Combinations}

The results obtained from the time kill assays indicated globally that all sub-fractions tested alone were fungistatic against all the microorganisms. On the contrary, the combinations exhibited fungicidal activity in most cases against the tested yeasts (Figure 1). Particularly, C. albicans and C. glabrata isolates were the most susceptible to the combinations $\mathrm{C} 36 / \mathrm{C} 2$ and $\mathrm{C} 36 / \mathrm{C} 12$, with all cells killed within 6 and 8 h of exposure, respectively. Comparatively, C. albicans NR-29450 was less sensitive to the combinations than the isolate. Overall, a fungicidal effect was obtained against the other microorganisms beyond $16 \mathrm{~h}$. Only C36/C12 showed fungicidal action against $C$. parapsilosis, and the two combinations were fungistatic on $C r$. neoformans.
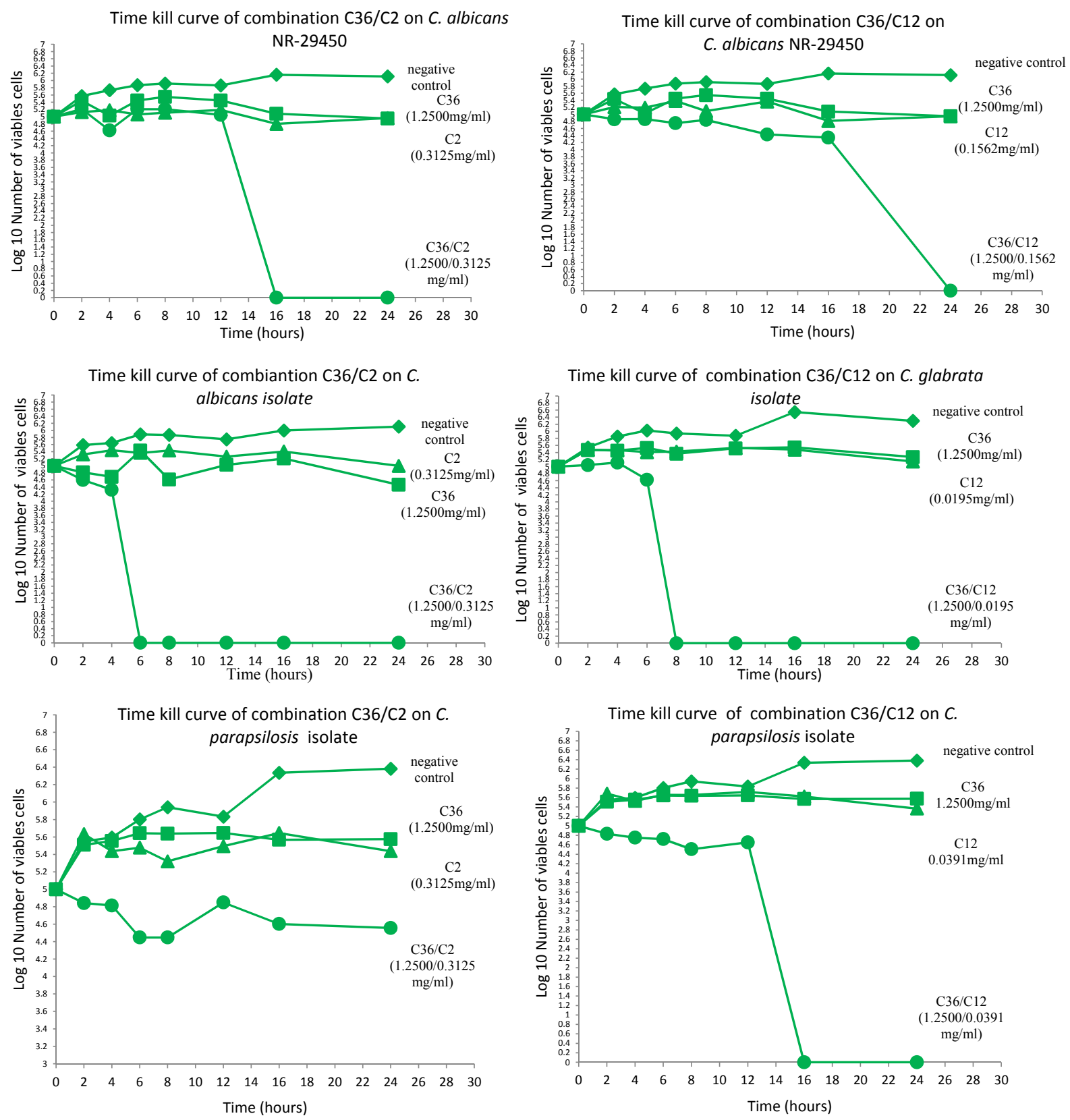

Figure 1. Cont. 

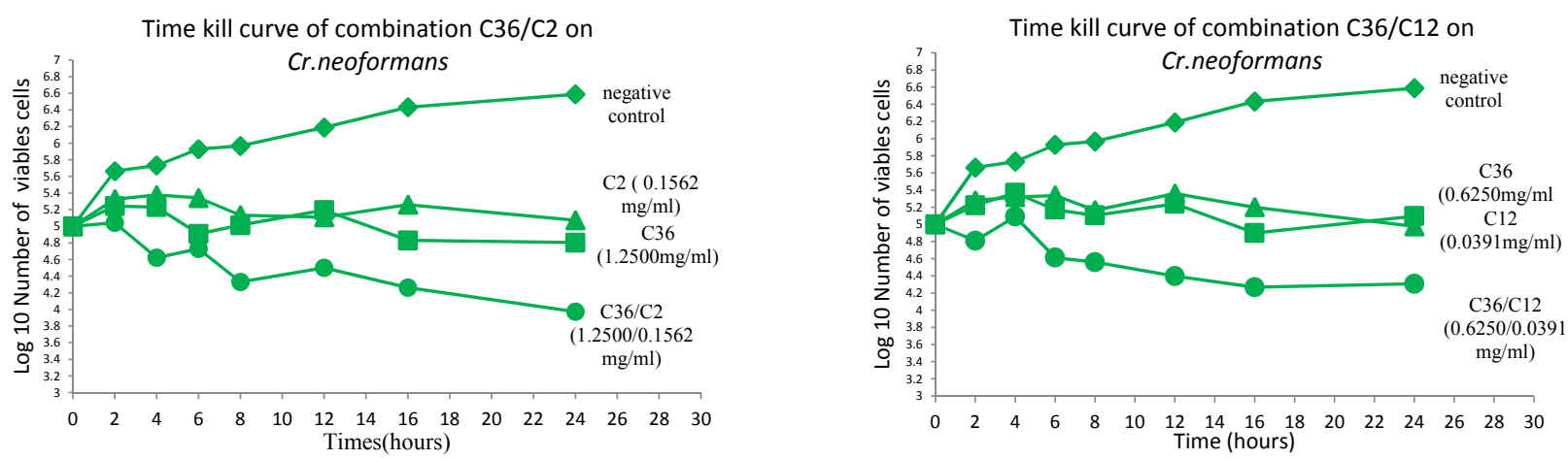

Figure 1. Time kill kinetic curves of combinations $C 36 / C 2$ and $C 36 / C 12$ against the tested yeasts. Sub-fractions were assessed alone and in combination for bioactivity at different time intervals.

\subsection{Phytochemical Screening of Crude Extracts and Selected Sub-Fractions}

The qualitative phytochemical screening of crude extracts and sub-fractions of interest showed the presence of alkaloids, flavonoids, tannins, saponins and steroids. However only two types of secondary metabolites (glucosides and steroids) were identified in all three most active sub-fractions (C2, C12, and C36), suggesting their implication in the exerted anti-yeast activity.

\section{Discussion}

The preparations of medicines by traditional healers are mainly carried out by boiling different organs of plants in water or macerating in alcoholic white wine. This information oriented the choice for extraction solvents. The antifungal activities obtained for T. catappa, T. mantaly and M. tenuifolia confirmed the traditional use of these plants in the treatment of fungal infections and also corroborated what had been previously found [39-41]. Aqueous and ethanolic extracts of stem bark of T. catappa and T. mantaly growing in the Cote d'Ivoire were shown to have anticandidal activity on clinical isolates of $C$. albicans with $\mathrm{IC}_{50}$ ranging from 0.02 to $0.55 \mathrm{mg} / \mathrm{mL}[39,40]$. Furthermore, a comparative study showed that extracts from $T$. mantaly were more active than those from $T$. catappa against Aspergillus fumigatus [40]. To the best of our knowledge, this is the first scientific report on the antifungal potential of M. tenuifolia extracts. Also, the present work is pioneering on the aspect of optimizing activities by coupling bio-guided fractionation and extracts combinations. Based on modern trends in combining drugs for activity optimization [34,42,43], combinations of $T$. mantaly and T. catappa with M. tenuifolia sub-fractions have considerably increased the anti-yeast activity. This optimized action of the combinations is more likely due to components of the sub-fractions acting synergistically against the pathogens $[33,44-46]$. Moreover, the simultaneous actions of metabolites on different targets enhance their bioactivity and might also reduce the advent of resistance by the fungi. Results from the time kill kinetics assays showed that combinations increased the antifungal activity and also changed the pharmacokinetics of extracts from fungistatic to fungicidal. This finding is particularly significant, given that among the drawbacks of azole drugs, they are rather fungistatic. This profile probably contributed to the development of resistance of clinical isolates from immunocompromised patients to azoles, given that residual yeast cells are not cleared, enabling a 
positive selection of drug-resistant mutants [47]. The qualitative phytochemical screening of the promising sub-fractions ( $\mathrm{C} 2, \mathrm{C} 12$ and $\mathrm{C} 36)$ led to the identification of steroids and glucosides as common classes of secondary metabolites. Steroids have been reported to have antibacterial and antifungal properties. The correlation between membrane lipids and sensitivity for steroidal compounds indicates the mechanism through which steroids specifically associate with membrane lipids and exert their action by causing leakages from liposomes [48]. Apart from this, steroidal glucosides were shown to possess potent antifungal activity [49]. Needed antifungal drugs should be ideally fungicidal. To achieve this, promising combinations identified within the framework of this study should be rationally studied in detail and potentially developed into novel drugs to control yeast infections.

\section{Conclusions}

The results achieved from this work indicate that T. mantaly, T. catappa and M. tenuifolia are potential sources of new antifungal drugs. The results also show improved potency in correlation with fractionation of extracts, as well as the combination of sub-fractions. The more promising combinations exerted synergistic and fungicidal actions against the tested yeasts. Overall, these findings support the continued investigation of extracts from the studied plants using the bio-guided fractionation and combination strategies to develop innovative therapies against mycoses.

\section{Acknowledgment}

The following reagent was obtained through BEI Resources, NIAID, NIH: C. albicans strain ATCC P37037. The authors thank the Yaoundé Central Hospital for providing the microorganisms. They also thank the Cameroon National Herbarium for the identification of plants.

\section{Authors Contributions}

F.F.B. and T.K.N. conceived and designed the work. T.K.N. participated in all manipulations, analyzed and interpreted the results and drafted the manuscript. C.M.J. and R.M.K.T. participated in plant collection, and antifungal and phytochemical tests. M.A.T.T. helped with bio-guided fractionation. E.M.Z., V.N., M.M. and S.B. read and critically revised the manuscript. All authors read and approved the final manuscript.

\section{Conflicts Interest}

The authors declare no competing interests

\section{References}

1. Sganga, G. Fungal infections in immunocompromised patients: Diagnosis, Therapy and Prophylaxis of Fungal Diseases. Mycoses 2011, 54, 1-3.

2. David, W.D.; William, W. Hope Therapy for fungal diseases: Opportunities and priorities. Trends Microbiol. 2010, 18, 195-204. 
3. Pemán, J.; Cantón, E.; Gobernado, M.; Spanish ECMM Working Group on Candidaemia. Epidemiology and antifungal susceptibility of Candida species isolated from blood: Results of a 2-year multicentre study in Spain. Eur. J. Clin. Microbiol. Infect. Dis. 2005, 24, 23-30.

4. Pfaller, M.A.; Diekema, D.J. Rare and emerging opportunistic fungal pathogens: Concern for resistance beyond Candida albicans and Aspergillus fumigatus. J. Clin. Microbiol. 2004, 42, 4419-4431.

5. Kumar, B.V.; Padshetty, N.S.; Bai, K.Y.; Rao, M.S. Prevalence of Candida in the Oral Cavity of Diabetic Subjects. Iran J. Microbiol. 2011, 53, 599-602.

6. Abu-Elteen, K.H.; Hamad, M.A.; Salah, S.A. Prevalence of Oral Candida Infections in Diabetic Patients. Bahr. Med. Bull. 2006, 28, 1-8.

7. Mlinariæ-Missoni, E.; Kaleniæ, S.; Vukelic, M.; de Syo, D.; Belicza, M.; Vaziæ-Babiæ, V. Candida infections of diabetic foot ulcers. Diabetol. Croat. 2005, 34, 29-35.

8. Park, B.J.; Wannemuehler, K.A.; Marston, B.J.; Govender, N.; Pappas, P.G.; Chiller, T.M. Estimation of the current global burden of cryptococcal meningitis among persons living with HIV/AIDS. AIDS 2009, 23, 525-530.

9. Lewis, R.E. Current Concepts in Antifungal Pharmacology. Mayo Clin. Proc. 2011, 86, 805-817.

10. De Almeid, O.P.; Scully, C. Fungal infections of the mouth. Braz. J. Oral Sci. 2002, 1, 19-26.

11. Laurent, M.; Gogly, B.; Tahmasebi, F.; Paillaud, E. Les candidoses oropharyngées des personnes âgées. Geriatr. Psychol. Neuropsychiatr. Vieil. 2011, 9, 21-28.

12. Magryś, A.; Kozioł-Montewka, M.; Starosławska, E.; Gabczyńska, B. The Prognostic and Diagnostic Markers of Invasive Candidiasis in Patients during Chemotherapy. Pol. J. Microbiol. 2005, 54, 207-213.

13. Fidel, P.L. History and update on host defence against vaginal candidiasis. Am. J. Reprod. Immunol. 2007, 57, 2-12.

14. Nelesh, G. HIV-associated opportunistic fungal infections: A guide to using the clinical microbiology laboratory. South. Afr. J. HIV Med. 2007, 1, 18-23.

15. Álvaro-Meca, A.; Jensen, J.; Micheloud, D.; Asunción, D.; Gurbindo, D.; Resino, S. Rate of candidiasis among HIV infected children in Spain in the era of highly active antiretroviral therapy (1997-2008). J. Infect. Dis 2013, 13, 1-15.

16. Brissaud, O.; Guichoux, J.; Harambat, J.; Tandonnet, O.; Zaoutis, T. Invasive fungal disease in PICU: Epidemiology and risk factors. Ann. Intensive Care 2012, 2, 6.

17. Pappas, P.G.; Kauffman, C.A.; Andes, D.; Benjamin, D.K.; Calandra, T.F.; Edwards, J.E.; Filler, S.G.; Fisher, J.F.; Kullberg, B-J.; Ostrosky-Zeichner, L.; et al. Clinical Practice Guidelines for the Management of Candidiasis: 2009 Update by the Infectious Diseases Society of America. Clin. Infect. Dis. 2009, 48, 503-535.

18. Lass-Flörl, C.; Mayr, A.; Perkhofer, S.; Hinterberger, G.; Hausdorfer, J.; Speth, C.; Fille, M. Activities of Antifungal Agents against Yeasts and Filamentous Fungi: Assessment according to the Methodology of the European Committee on Antimicrobial Susceptibility Testing. Antimicrob. Agents Chemother. 2008, 52, 3637-3641.

19. Ruan, E.; Kock, J.L.F.; Pohl, C.H. Candida albicans or Candida dubliniensis? Mycoses 2009, 54, 1-16. 
20. Busari, O.; Adeyemi, A.; Agboola, S. Cryptococcal Meningitis in AIDS. Internet J. Infect. Dis. 2009, 7, 1. Available online: http://print.ispub.com/api/0/ispub-article/8148 (accessed on 18 August 2015).

21. Maslin, J.; Morand, J.J.; Menard, G.; Camparo, P. Les Cryptococcoses. Med. Trop. 2002, 62, 480-484.

22. Millogo, A.; Ki-Zerbo, G.A.; Andonaba, J.B.; Lankoandé, D.; Sawadogo, A.; Yaméogo, I.; Sawadogo, A.B. La cryptococcose neuroméningée au cours de l'infection par le VIH au Centre hospitalier de Bobo-Dioulasso (Burkina Faso). Bull. Soc. Pathol. Exot. 2004, 97, 119-121.

23. Petrikkos, G.; Skiada, A. Recent advances in antifungal chemotherapy. Int. J. Antimicrob. Agents 2007, 30, 108-117.

24. Kanafani, Z.A.; Perfect, J.R. Antimicrobial resistance: Resistance to antifungal agents: Mechanisms and clinical impact. Clin. Infect. Dis. 2008, 46, 120-128.

25. Tscherner, M.; Schwarzmüller, T.; Kuchler, K. Pathogenesis and Antifungal Drug Resistance of the Human Fungal Pathogen Candida glabrata. Pharmaceuticals 2011, 4, 169-186.

26. Neelavathi, P.; Venkatalakshmi, P.; Brindha, P. Antibacterial activities of aqueous and ethanolic extracts of Terminalia catappa leaves and bark against some pathogenic bacteria. Int. J. Pharm. Pharm. Sci. 2013, 5, 114-120.

27. Neuwinger, H.D. African Traditional Medicine, A Dictionary of Plant Use and Applications; Medpharm Scientific: Stuttgart, Germany, 2000.

28. Therese, K.L.; Bagyalakshmi, R.; Madhavan, H.N.; Deepa, P. In vitro susceptibility testing by agar dilution method to determine the minimum inhibitory concentrations of amphotericin B, fluconazole and ketoconazole against ocular fungal isolates. Ind. J. Med. Microbiol. 2006, 24, 273-279.

29. NCCLS. Reference Method for Antifungal Disk Diffusion Susceptibility Testing of Yeasts; Approved guideline; NCCLS document M44-A2; National Committee for Clinical Laboratory Standards: Wayne, PA, USA, 2004.

30. Clinical and Laboratory Standards Institute (CLSI). Reference Method for Broth Dilution Antifungal Susceptibility Testing of Yeasts, 3rd ed.; CLSI: Wayne, PA, USA, 2008.

31. Iten, F.; Saller, R.; Abel, G.; Reichling, J. Additive antimicrobial effects of the active components of the essential oil of Thymus vulgaris-chemotype carvacrol. Planta Med. 2009, 75, 1231-1236.

32. Berenbaum, M.C. A method for testing for synergy with any number of agents. J. Infect. Dis. 1978, 137, 122-130.

33. Van Vuuren, S.; Viljoen, A. Plant-Based Antimicrobial Studies Methods and Approaches to Study the Interaction between Natural Products. Planta Med. 2011, 77, 1168-1182.

34. Klepser, M.E.; Ernst, E.J.; Lewis, R.E.; Ernst, M.E.; Pfaller, M.A. Influence of test conditions on antifungal time-kill curve results: Proposal for standardized methods. Antimicrob. Agents Chemother. 1998, 42, 1207-1212.

35. Aromedee, C.; Wichitchote, P.; Jantakun, N. Spectrophotometric Determination of Total Lactones in Andrographis paniculata Nees. Songklanakarin J. Sci Technol. 2005, 27, 1227-1231.

36. Odebeyi, O.O.; Sofowara, F.H. Antimicrobial alkaloids from a Nigerian Chewing Stick (Fagara zanthoxyloides). Planta Med. 1978, 36, 204-207. 
37. Sofowara, A. Medicinal Plants and Traditional Medicine in Africa, 2nd ed.; Spectrum Books Limited: Ibadan, Nigeria, 1993.

38. Trease, G.E.; Evans, W.C. Pharmacognosy, 13th ed.; Bailliere Tindall: London, UK, 1989.

39. Ackah, J.A.B.; Kra, A.K.M.; Zirihi, G.N.; Guede-Guina, F. Évaluation et essais d'optimisations de l'activité anticandidosique de Terminalia catappa Linné (tekam3), un extrait de Combretaceae de la pharmacopée ivoirienne. Bull. Soc. R. Sci. Liège 2008, 77, 120-136.

40. Yapi, G.Y.; Adou, K.M.; Ackah, J.A.B.; Djaman, A.J. Évaluation de l'activité antifongique et essai de purification des principes actifs des extraits de Terminalia mantaly (H Perrier), une eaCombretaceae, sur la croissance in vitro de Candida albicans. Bull. Soc. R. Sci. Liège 2011, 80, 953-964.

41. Zirihi, G.N.; N'guessan, K.; Kassy, N.J.; Coulibaly, K.; Djaman, A.J. Evaluation and comparison of antifungal activities of Terminalia catappa and Terminalia mantaly (Combretaceae) on the in vitro growth of Aspergillus fumigatus. J. Med. Plant Res. 2012, 6, 2299-2308.

42. Lewis, R.E.; Diekema, D.J.; Messer, S.A.; Pfaller, M.A.; Klepser, M.E. Comparison of E-test, chequerboard dilution and time-kill studies for the detection of synergy or antagonism between antifungal agents tested against Candida species. J. Antimicrob. Chemother. 2002, 49, 345-351.

43. Lin, Y.T.; Labbe, R.G.; Shetty, K. Inhibition of Vibrio parahaemolyticusin seafood systems using oregano and cranberry phytochemical synergies and lactic acid. Innovat. Food Sci. Emerg. Technol. 2005, 6, 453-458.

44. Nwodo, U.U.; Ngene, A.A.; Iroegbu, C.U. Effects of Fractionation on Antibacterial Activity of Crude Extracts of Tamarindus indica. Afr. J. Biotechnol. 2010, 9, 7108-7113.

45. Ncube, B.; Finnie, J.F.; van Staden, J. In vitro antimicrobial synergism within plant extracts combinations from three South African medicinal bulbs. J. Ethnopharmacol. 2012, 139, 81-89.

46. Onyewu, C.; Blankenship, J.R.; del Poeta, M.; Heitman, J. Ergosterol biosynthesis inhibitors become fungicidal when combined with calcineurin inhibitors against Candida albicans, Candida glabrata, and Candida krusei. Antimicrob. Agents Chemother. 2003, 47, 956-964.

47. Shimada, T. Salivary proteins as a defence against dietary tannins. J. Chem. Ecol. 2006, 32, 1149-1163.

48. Raquel, F.E. Bacterial lipid composition and the antimicrobial efficacy of cationic steroid compounds. Biochim. Biophys. Acta 2007, 1768, 2500-2509.

49. Ahsan, S.; Ejaz, A.; Abdul, M. Recurvosides A and B, Antifungal Novel Steroidal Glucosides from Haloxylon recurvum. Z. Naturforsch. B. 2006, 61(9), 1148-1152.

(C) 2015 by the authors; licensee MDPI, Basel, Switzerland. This article is an open access article distributed under the terms and conditions of the Creative Commons Attribution license (http://creativecommons.org/licenses/by/4.0/). 